$\underline{\text { Review Article }}$

\title{
An Ethical Reflection on the Heritability Argument in the Germline Genetic Intervention Debate
}

Maide Baris ${ }^{1}$

\begin{abstract}
$\underline{\text { Abstract }}$
Germline genetic intervention (GGI) has been one of the most discussed topics within the bioethics literature since 2012, when the programming of CRISPR/Cas9 for a specifically targeted gene region has become possible. While some authors are optimistic about what GGI may offer, others strongly disagree and refute the use of this technology for different reasons. This paper will aim to examine one of the most widespread arguments against GGI, namely "heritability" argument, comprehensively. Firstly, it will aim to examine the moral importance of the germline. Secondly, it will try to understand three possible assumptions of the heritability argument. Then it will try to respond to these assumptions and argue that they are neither scientifically supportable nor rationally solid for rejecting GGI altogether.
\end{abstract}

Keywords: Genethics, Germline Genetic Intervention, Heritable Genome Editing, Heritability, Bioethics.

International Journal of Human and Health Sciences Vol. 05 No. 04 October'21 Page : 405-411 DOI: http://dx.doi.org/10.31344/ijhhs.v5i4.349

\section{Introduction}

Long awaited and debated, germline genetic interventions (GGI) are at the door of reproductive clinics. With the He Jiankui affair (2018), the GGI debate has been fired up once again. The He Jiankui affair has resulted in an increased awareness not only about the necessity of regulatory arrangements, but also about the great difficulties in building regulation or imposing a ban that is recognized from an international perspective. When He's study was examined, serious omissions regarding such regulations as consent forms, ethics committee approval, supervision, transparency, and safety of the procedure were found ${ }^{1}$.

Even before the He Jiankui affair, many authors have been arguing that germline genetic intervention (GGI) is ethically more contentious than somatic genetic intervention (SGI) because of its heritability to the future generations ${ }^{2,3}$. They believe that there is a moral dividing line between GGI and SGI, that is the "heritability of the GGI",3.4. This argument is widely accepted and supports a universal condemnation of GGI, as well as universal acceptance of SGI, based on the "mortal soma" versus "immortal germline" distinction. This is partly because during the discussions about genetic research in the 1970s, it was concluded that SGI was a form of conventional therapy. After these discussions, the distinction between SGI and GGI was drawn so that the former has been widely considered morally acceptable while the latter is a highly contentious matter ${ }^{5}$. Cavaliere also reasserts that while many authors consider SGI as a continuation of conventional medicine, most deem GGI as unconventional and unprecedented ${ }^{6}$. As Rehmann-Sutter once pointed, most of the metaphysics-based objections raised against GGI stem from different evaluations of the germline phenomenology ${ }^{7}$. These differences in evaluation are based on the conviction that GGI will have some important ethical implications for human beings. When the genethics literature is widely reviewed, one can notice that alleged moral importance of the "germline" seems to have three different assumptions. The first assumption seems to suggest that the germline genome is an "unchangeable" entity that is stable and immune to changes. The second one seems to imply that heritability is specific to GGI and there is no other means to change the germline genome that travels between generations. The third assumption seems to hold that protecting the human germline is akin to protecting human beings as their essence lies within their genome, thus we have a moral duty to protect the human genome as it is. This paper will firstly elaborate the alleged moral importance of the germline, and then will analyze and reflect on these three possible different but interrelated assumptions underlying the heritability argument. Next, it will try to respond to the claims of these assumptions that support the heritability argument and argue that they are neither strong as they claim

Correspondence to: Maide Barış, Istanbul University, Graduate School of Health Sciences; Istanbul Health and Technology University, Medical Faculty, Medical History and Ethics Department. E-mail: baris.maide@gmail.com 
to be nor adequate for rejecting GGI altogether on the grounds of "heritability".

From the beginning, I should make it clear that no one shall defend clinical application of GGI unless it becomes sufficiently safe and legal. Therefore, this paper is not going to discuss the safety or legality of heritable genetic interventions, it only discusses the notion of heritability in terms of GGI and how this notion is used to refute the use of this technology. Besides, this paper will not also go into the terrain of treatment-enhancement dichotomy that also holds a wide space in the genethics debate.

\section{Moral Importance of Germline: Misunderstanding or Overvaluation?}

The germline can be viewed as a lasting connection between successive generations. Gamete cells as the transmitters of the germline genome, represent the physical reality of this intergenerational relationship ${ }^{8}$. In this context, gamete cells both play a biological role and have a social importance by providing a relational link between generations. Before August Weismann introduced the "germline" concept, interaction between preceding and subsequent generations was thought to occur only through cultural, social, and ecological interactions. However, the discovery of germline within the gamete cells has added the "biological dimension" to these existing interactions. Although individuals manage to isolate themselves from the cultural, social, and ecological interaction elements they inherited from previous generations, they cannot isolate themselves from the elements of biological interaction they inherited through the "germline". Therefore, according to some authors, this connection established with the germline is much more personal and corporeal than the other intergenerational interactions because the biological interaction provided by the continuity of the germline is somehow related to morphological, structural, and functional capabilities of our body, to who we are, Moreover, this link is generative, as changes in the genetic composition of gamete cells may affect those capabilities of future generations to a certain extent. Thus, the importance of the germline seems to be that it physically (or maybe better if said "in a molecular" or "in a subcellular level") connects one generation to the next. In this context, GGI seems to be accused of causing changes in the productive/generative structure of those effects that are passed down between generations ${ }^{7}$.

So, what is the moral significance of this generativity, knowledge and connection embodied in the germline genome for us? Why does a germline genetic intervention worry us more than changing one of the components in our cultural, social or ecological interactions? Is it a kind of hidden "genetic exceptionalism" that claims the alleged exceptional difference of genetic information? Or is it something else? By saying "any heritable change in the genome is morally unacceptable", what does heritability argument claim? We should go beyond the lines and try to understand the moral reasoning of the heritability argument. To do so, we will review three possible assumptions of the argument.

\section{$1^{\text {st }}$ Assumption of Heritability Argument:} "Germline genome is stable and does not change in the absence of an intentional intervention"

First assumption of the heritability argument seems to presume that the germline genome is immune to any changes, and it is a protected entity within a shell so that it passes down to the future generations without any change. On the other hand, GGI threatens this protection by altering it. Based on this assumption, heritability argument may claim:

- Germline genome is immune to any changes.

- GGI causes a change in the germline genome.

- If something causes a change in the germline genome, it is morally wrong and impermissible.

- Then, GGI is morally wrong.

The first assumption that might support the heritability argument against the GGI, seems largely to feed on a relatively outdated view of the genome. According to this outdated view of genome, the genome was mainly thought of as an everlasting entity, and later as a stable set of genes that influence/determine the developmental processes of the organism; although it has been known that mutations can be accumulated in DNA over time and the genome (including the germline genome) is shaped by the evolution itself. Today, the genome is no longer thought as a defined set of genes that an individual has had from birth. Rather, it is considered as a plastic and evolving phenomenon whose composition and functioning can change significantly throughout an individual's lifetime ${ }^{9}$. It is now a well-established fact that the genome is more than a group of genes at the level of an individual organism, and the structure and functioning of this system is a more complex and dynamic issue. Therefore, which regions of the genome would be expressed, whether this expression would yield a product, or what the expressed products would look like are determined not only by certain genes but also by a multidimensional operating system and process. This updated view of the genome suggests that 
the genome could have never been a timeless or stable entity. On the contrary, the genome has been always changing, dynamic and reactive to the surroundings; and, can neither be limited nor preserved in spatial and temporal terms $\mathrm{s}^{10,11}$.

To recap, the human genome is a reactive, plastic and dynamic entity, adopting a pluralistic system of governance that includes intracellular, intercellular, and even environmental processes outside the organism. Besides, because the gene of the reactive genome had never been stand-alone administrative unit or entity as it was thought before, behavior of the multi-component genome does not follow a fixed destiny, but changes throughout a person's lifetime. This also implies that the environmental factors such as nutrition, exercise, smoking habits, etc. have now become an important element that define what a genome looks like and how it behaves ${ }^{9,10,11}$.

Now, it is time to ask that what impact has the evolution of the stable and timeless genome towards the reactive genome may have on the "germline" phenomenology? This is a very important question not only because this new understanding of the genome has important methodological implications for biological and biomedical research, but also has significant ethical implications for GGI debate. It seems that objecting the GGI because of "heritability" or "meddling into the stable germline genome" does not constitute a valid and sufficient ethical argument on its own, since the concept of reactive genome suggests that germline genome that is inheritable to the offspring is not a stable entity but prone to multidimensional changes that epigenetic studies well documented. All in all, truth of the postgenomic era- that is "the human genome is in constant change and transformation in a multiple and simultaneous processes and has a dynamic and plastic structure which has never been stable or immune to changes"-, renders the first possible assumption of heritability argument ineffective $e^{8,9,10,11}$.

\section{$2^{\text {nd }}$ Assumption of Heritability Argument: "Heritability is specific to GGI"}

Second assumption of the heritability argument (based on the first assumption) seems to imply that impact of heritability is specific to GGI. In a similar vein with the first assumption, the second one presumes that the germline genome is stable and immune to changes; and also, it defends that GGI is the only procedure that can change it.

According to this version, heritability argument may claim:

- Germline genome is immune to any changes and heritable (i.e. passes down to the future generations as it is.)

- Nothing but only GGI can change the germline genome.

- If something causes a change in the germline genome, it is morally wrong and impermissible because it is heritable.

- Then, GGI is morally wrong.

The second assumption seems to limit the heritability to the GGI particularly. Here, "heritability" appears as a phenomenon passed down between generations. In other words, what makes GGI problematic is its heritability, that is, it interferes with biological interaction by creating an effect that will be passed on between generations. However, "germline genetic interventions" are not the only incidents causing transgenerational inheritance. Many things, from agriculture to animal husbandry, from education to city planning, from nutrition to sports, from public health measures to cultural habits, may have transgenerational impacts ${ }^{1,8,12}$.

Moreover, there are some mechanisms that have been proven by scientific studies and can be passed on to future generations, even though they do not involve a direct intervention to the germline or even have a genetic basis. As an example of biological and non-genetic mechanism that impacts on future generations' genome, the biology of reproduction can be given. Gamete cells belonging to female and male during reproduction - in reproductive organs or in in vitro test tubes - contain much more than the germline genome. Gamete cells are carried along with some enzymes and secretions found in the male seminal and female oviductal fluid. Experimental studies have shown that these enzymes affect the offspring to be born by a nongenetic mechanism and these effects are passed on to the next generation ${ }^{13}$. Moreover, it has also been shown that the effects of some variations induced by epigenetic mechanisms on germ cells are transferred to the offspring ${ }^{14}$. For example, studies on the effects of the Dutch Hunger Winter period during the Second World War show that some epigenetic changes that occur due to the mother's hunger during early pregnancy affect the early embryo in the womb in a way that causes variation in the germline and thus the generations. It has shown that it can provide a heritable effect transferred between generations. Similarly, studies included inhabitants of Överkalix, their children and grandchildren in the northern Swedish city, who experienced irregular famine during WW II, showed that grandparents' nutrition just before 
puberty was associated with transgenerational (gender-specific) phenotypes ${ }^{15}$. Therefore, objecting the GGI because of "heritability" or "affecting future generations" does not constitute a valid and sufficient ethical argument on its own, since it is not an exceptional case specific to germline genetic interventions.

In response to the non-exclusiveness of GGI in terms of the heritability, there are some authors who claim GGI is "too risky" as a heritable procedure, in comparison to other transgenerational effectcreating incidents, that will have negative impact on not only the person who undergone it but also future generations in case of technical or procedural failure. One could not ignore such a serious implication. Yet again one could also equally pay attention to, for instance, human nutrition status all around the globe to be consistent as Överkalix or Dutch Hunger incidents prove that human nutrition may have heritable impact; or otherwise demonstrate the "exceptionality" of GGI, to be consistent in ethical argumentation.

Furthermore, apart from the allegedly "rare or dubious cases" mentioned above, the human germline genome is constantly exposed to mutation, many daily and routine activities of human beings affect the likelihood of mutations occurring ${ }^{1}$. For example, delaying fatherhood to a later age increases the number of mutations in the sperm, and these mutations are passed on to later generations. According to Gyngell et al., many authors who adopt the heritability argument do not think that such inherited mutations pose a serious moral risk for future generations ${ }^{12}$. But if they want to be consistent, they should be expected to advocate regulations, such as screening for the sperm of those who want to become fathers over a certain age.

The fact that a heritable variation that is caused by a random mutation or an intervention performed by GGI seems to make a difference for the heritability argument. However if the heritability argument claims that there is a difference between these two, it should based on solid ethical arguments and be free of fallacies of genetic determinism ${ }^{12}$.

Moreover, like spontaneous mutations, some cancer treatments potentially cause permanent and heritable changes/mutations in the germline genome. However, due to possible permanent and heritable changes in the genome of people who have undergone cancer treatment, it is not thought or defended that a preliminary screening of gamete cells should be required to be able to use reproductive technologies. In response to these arguments, some authors contend that "it already occurs" or "we have always done it" arguments do not imply that we should continue to do it by any means possible 5 . At this point, one should be careful with a hidden form of both genetic exceptionalism (i.e. the genetic information is exceptionally different other form of information) and genetic determinism (i.e. humans' traits, actions, and preferences are predetermined -to a varying degree- by their genes), exist in these arguments, that cannot demonstrate the exclusivity or exceptionality of the GGI in a reasonable way. To recap, although the second assumption of the heritability argument, like the first and third ones, seems to focus mainly on the "heritable changes" that GGI causes; it blames GGI as the only possible cause of heritable changes and ignores the abovementioned (old age fathering, cancer treatments, nutritional status, etc.) causes. Thus, unless the authors who argue against GGI because it causes heritable changes explain why GGI is exceptionally or exclusively objected, the argument does not seem defensible from an ethical point of view.

$3^{\text {rd }}$ Assumption of Heritability Argument: "We have a moral duty to protect the human genome as it is"

The third assumption underneath the heritability argument seems to presume that protecting the human germline genome is akin to protecting human beings at the species level, as their essence lies within the genome. Sounds more like an essentialist argument, this version argues that human genome is the common heritage of the humanity and should be protected for the sake of humanity ${ }^{16}$.

According to this version, heritability argument claims:

- The human (germline) genome is the common heritage of the humanity.

- If something causes a change in the germline genome, it is morally wrong and impermissible because it targets the humanity.

- GGI causes a change in the germline genome.

- Then, GGI is morally wrong.

Universal Declaration on the Human Genome and Human Rights, published by UNESCO (November 11, 1997) endorses "the preservation of the human genome as common heritage of humanity"17. John Harris criticizes this line of thought by stating that " $[\mathrm{t}]$ hose who appeal to the common heritage of humanity in this way see the present evolved state of the human genome as a state that must be "frozen," as far as is possible and in perpetuity, at this particular evolutionary stage'. This is a scientific fact that all human 
beings are "the products of a germline-altering process called evolution" through meiotic processes in sexual reproduction ${ }^{18}$. At this point, a proponent of the third assumption that supports the heritability argument may claim that GGI is likely to be more dangerous than "unintentional genetic modification"- that is, the process of mutation, inheritance, and selection that occurs whether we like it or not. As a matter of fact, random genetic mutations may be 'more likely to be deleterious than beneficial, but this is not necessarily true of targeted modifications designed with some understanding of their likely effects' ${ }^{\prime}$.

Nevertheless, there are authors who insist that the influence of GGI on heritability is important and is not akin to any other mechanisms causing a change in the germline genome. They claim that because GGI is planned and aimed at a specific gene or sequence, its impact in heritability is not contingent like abovementioned mechanism, but more likely deterministic. This argument claims that other phenomena (nutrition, war, agriculture, lifestyle, age, etc.) that affect the future generations are contingent in nature, while GGI is an intentional manipulation. From a different perspective many authors argue that GGI causes heritable changes that will be passed down to our future offspring and we cannot approve GGI because we cannot create such an effect to the future generations without their consent. Here, I am intentionally avoiding this "consent" argument to concentrate mainly on the heritability argument.

From a scientific point of view, the truth is that the human gene pool cannot be contained to be preserved as it has no bottom, no top, or no side walls ${ }^{20}$. On the contrary, evolution studies have shown that the human genome has been never "one and only" or stable. As a matter of fact, it is more appropriate to talk about "human pangenome" suggesting that genomes belong to humans are too many, not "human genome" implying that it is a singular entity. Besides, studies have also shown that the genome can never be "preserved" or "protected". Evolution itself has never been entirely contingent since Homo sapiens first appeared, as they have always made a choice (plantation, domestication, wars, etc.). Referring to "the human nature" as a singular phenomenon has transformed into "protecting the contingency" which neither needs nor demands any protection by definition. It is going to be contingent eventually whether GGI is performed or not. Therefore, refuting GGI based on the third assumption of the heritability argument also do not seem sound from an ethical perspective.

\section{Discussion}

The scientific image of the genome has changed quite rapidly over the last two decades and is still changing. This change also affects our understanding of how we should assess the risks of GGI practices. Ensuring the safety of a genetic intervention in a postgenomic world means not only following the entire life cycle of the organism being intervened, but also examining and even controlling the interactions of this organism with its environment. However, some authors who oppose GGI do not intend to restrain the effects of the environment with the same attention they insist to have on genetic factors, thus accepting the superiority of genetics in the so-called naturenurture (or genetics-environment) distinction.

Genes are not concrete units, but constantly interact with their internal and external environments. This fact states that genes are units that cannot be isolated, and that all the nucleotide sequences found on DNA are somehow connected with each other and with their environment. Based on this concept of the reactive genome, this fact provides a sufficient reason for not allowing germline genetic interventions, according to some authors $^{2,3}$. However, I find this interpretation rather contradictory. Because, since genes, noncoding DNA sequences and the internal-external environment are involved in such an intertwined process that cannot be separated from each other with clear lines, why shall we focus on the possible impact of GGI but ignore the impact of our daily life conditions such as nutrition or chemotherapy on the genome? Why don't we oppose other nongenetic multidimensional and unexpected effects of the environment to the same extent, as we now know that our genomes are reactive and dynamic; susceptible to both intracellular, intercellular, or environmental factors? Because if the genome is a holistic structure and if the internal and external dynamics are integrated, why should we emphasize only the possible negative effects of genetic intervention and ignore other factors? Focusing only on the genome and a very outdated understanding of the gene, may negatively impact our ethical judgements about the GGI. For this reason, ethical arguments should be familiar with the postgenomic concepts and understanding of the genetics to avoid commonly committed fallacies, such as genetic exceptionalism and genetic determinism.

Studies conducted in the postgenomic period have shown that the intervention on the germline genome is not only through genetic intervention, but also the internal and external environment is 
significantly effective. Interestingly, this result seems to benefit both those who defend GGI and those who oppose it. Advocates of GGI claim that many non-genetic factors change the biological interaction between generations by changing the germline. Therefore, those in this group consider attaching extra-importance to genetic interventions as a reflection of genetic exceptionalism. On the other hand, this dynamic and reactive genome model of the postgenomic period strengthens the hand of GGI opponents who argue that the effects of the interventions cannot be predicted. Both sides underline the shortcomings of the genetics paradigm, making use of epigenetics, which is the main representative and contributor of the postgenomic era ${ }^{8,9}$.

\section{Conclusion}

In conclusion, it is possible to argue that postgenomic research has made an important transformation in germline phenomenology and our understanding of "heritability". Within the limits of "genetics of the $20^{\text {th }}$ century", "genes" had been viewed as the stable units of human genome which was immune to any internal and external effect. Thus, scientists along with social experts had seen "genes as they are" as "essential" to the existence of human beings. This outdated image of genome was fragile. However, within the postgenomic era, with the contributions of epigenetic studies, we come to understand that genome is not a stable entity, and it is in a constant relation with its internal and external environment. The new genome is dynamic, plastic and robust so that it can endure various challenges. Thus, the postgenomic period transformed our perception of the germline by including the biological and hereditary contributions of the interactions of the organism with its internal and external environment and eliminated the image of the genes considered as the only intergenerational element of biological interaction. This transformation will make it possible to question the material and spiritual meanings attributed to the germline. Our understanding of the germline has changed and the monocratic dominance of this embodied knowledge for our lives has been challenged. Thus, we should also challenge "heritability argument" in GGI debate with our new knowledge about the genome.

To sum up, it appears that GGI is not as exceptional in its impact on the genome, as the heritability argument may have presumed. According to many authors who criticize the heritability argument, ethical debates about GGI should follow current scientific developments and renew and rearrange their arguments ${ }^{1,8,12}$. Because, from a moral point of view, the question of which method causes heritable changes, in other words, whether genetic change is caused by technologies or other nongenetic (environmental, cultural, psychological or physiological) factors, remains unrelated and unfounded as long as the person cannot show the moral exceptionalism of genetic intervention technologies.

Conflict of Interest: None declared.

Ethical Approval Issue: No ethical approval was required for this study.

Funding Statement: This study received no specific funding. 


\section{References}

1. Greely HT. Human Germline Genome Editing: An Assessment. The CRISPR Journal. 2019;2(5):253265.

2. Caplan AL, Parent B, Shen M, Plunkett C. No time to waste - the ethical challenges created by CRISPR. EMBO Reports. 2015;16(11):1421-6.

3. Baylis F. 'Broad societal consensus' on human germline editing. Harvard Health Policy Review. 2016;15(2):19-22.

4. de Miguel Beriain I. Should human germ line editing be allowed? Some suggestions on the basis of the existing regulatory framework. Bioethics. 2019;33(1):105-11.

5. Baylis F, Robert J. Radical rupture: exploring biological sequelae of volitional inheritable genetic modification In Rasko, John E.J.; O'Sullivan, Gabrielle M.; Ankeny, Rachel A., eds. The Ethics of Inheritable Genetic Modification: A Dividing Line? Cambridge: Cambridge University Press. 2006:13148.

6. Cavaliere G. The Ethics of Human Genome Editing: Background Paper for WHO Expert Advisory Committee on Developing Global Standards for Governance and Oversight of Human Genome Editing. 2019.

7. Rehmann-Sutter C. Why human germline editing is more problematic than selecting between embryos: Ethically considering intergenerational relationships. The New Bioethics. 2018;24(1):9-25.

8. Lewens T. Blurring the germline: Genome editing and transgenerational epigenetic inheritance. Bioethics. 2020;34(1):7-15.

9. Guttinger S. Editing the reactive genome: towards a postgenomic ethics of germline editing. Journal of Applied Philosophy. 2020;37(1):58-72.

10. Keller EF. The century of the gene. Harvard University Press. 2009.

11. Jablonka E, Lamb MJ. Evolution in four dimensions, revised edition: Genetic, epigenetic, behavioral, and symbolic variation in the history of life. MIT press. 2014.

12. Gyngell C, Douglas T, Savulescu J. The ethics of germline gene editing. Journal of Applied Philosophy. 2017;34(4):498-513.

13. Bohacek J, Mansuy I. Molecular insights into transgenerational non $\square$ genetic inheritance of acquired behaviours. Nat. Rev. Genet. 2015;16(11):641-52.

14. Gapp K, Bohacek J. Epigenetic germline inheritance in mammals: Looking to the past to understand the future. Genes, Brain Behav. 2018;17:1-12.

15. Lassi M, Teperino R. Introduction to Epigenetic Inheritance: Definition, Mechanisms, Implications and Relevance. In Beyond Our Genes, Springer, Cham. 2020:159-173.

16. Annas G, Andrews L, Isasi R. Protecting the Endangered Human: Toward an International Treaty Prohibiting Cloning and Inheritable Alterations. American Journal of Law \& Medicine. 2002;28(23):151-178.

17. UNESCO (1997). Universal declaration on the human genome and human rights. RefWorld. Available at: http://www.refworld.org/docid/404226144.html Accessed 01.07.2021

18. Harris J. Germline manipulation and our future worlds. The American Journal of Bioethics. 2015;15(12):30-34.

19. Chan S. Playing it Safe? Precaution, Risk, and Responsibility in Human Genome Editing. Perspectives in Biology and Medicine. 2020;63(1):111-125.

20. Juengst ET. “Alter-ing” the human species? Misplaced essentialism in science policy. In Rasko, John E.J.; O'Sullivan, Gabrielle M.; Ankeny, Rachel A., eds. The Ethics of Inheritable Genetic Modification: A Dividing Line? Cambridge: Cambridge University Press. 2006:149-58. 